\title{
Application of Image Processing in the Medieval Mosaic Conservation
}

\author{
Barbara Zitová, Jan Flusser and Filip Šroubek
}

\begin{abstract}
Department of Image Processing, Institute of Information Theory and Automation, Academy of Sciences of the Czech Republic, Pod vodárenskou věží 4, 18208 Praha 8, Czech Republic
\end{abstract}

\begin{abstract}
We present an application of digital image processing for an analysis of medieval mosaic conservation. The reconstructed art piece was "The Last Judgment" mosaic, situated on the wall of the St. Vitus cathedral in Prague, Czech Republic. The historical photograph of the mosaic from the $19^{\text {th }}$ century was compared with the photograph of the current state. The images were firstly preprocessed to increase their quality (noise reduction, deblurring). In the second stage, geometrical differences between images were removed by means of image registration techniques - mutual information and feature point correspondence. Finally, differences of the current and historical photographs were identified.
\end{abstract}

Keywords: art conservation, image restoration, minimization methods, image registration, mutual information.

\section{INTRODUCTION}

Our paper demonstrates an application of digital image processing for the analysis of the medieval mosaic conservation. The splendid "The Last Judgment" mosaic is situated on the outer wall of the St. Vitus cathedral in the premise of Prague Castle, Czech Republic (Fig. 1). It is made of almost 1000000 glass cubes and it was completed in 1371, under the reign of King Charles IV.

The dilapidation of the mosaic during the centuries was very severe, due to the high amount of agents contained in the glass, due to temperature fluctuations (the measured range of the temperature is from -28 to +60 degrees of Celsius) and, recently, due to air pollution. Large amount of effort has been put to conserve and protect the mosaic. The last attempt started in 1992, when the U.S. Getty Conservation Institute jointly with Czech specialists reconstructed the whole mosaic. The renovation was finished in September 2000.

During the last conservation, an historical black and white photograph of the mosaic was discovered in the archives. It was taken on a glass plate by Jindrich Eckert, the famous Czech photographer, in 1879. This photograph captures the mosaic before its removal due to a great storm damage in 1890 (the renewed mosaic was returned to the cathedral wall in 1910). This photo gives us an unique opportunity to compare the current state of the mosaic with the mosaic state at the end of the $19^{\text {th }}$ century.

The aim of our work was to reveal original patterns, captured on the historical photograph, which already disappeared from the mosaic and which are not apparent on the photograph due to the high level of present noise and blurring. Moreover, we were asked to evaluate the mosaic reconstructions realized on the mosaic since 1879.

For our experiments, we used the image of the current mosaic state taken by a digital camera (see Fig. 1 bottom) and the digitized negative (1200 DPI) of Eckert's photograph (see Fig. 1 top). The special tonality of the historical image is caused by the low sensitivity of the employed photographic material to the red part of the visible spectra. The photograph has a high level of noise and blurring caused by various factors such as aging, chemical changes of the photo material and wrong focus of the camera. The current photograph, depicting approximately the same parts of the mosaic as the historical image, was transformed to the grayscale space. For the presentation, achieved results of image restoration are shown on two severely blurred areas $(450 \times 300$ and $300 \times 400$, see Fig. 2). Image registration results are demonstrated on two large mosaic parts, the Resurrection scene (see Fig. 4) and the central part with Jesus Christ (see Fig. 5).

Section 2 describes the image restoration process applied primarily on the historical image. The restoration was necessary because of the degradation of the photograph (noise, blurring, etc.). Section 3 provides information about used image registration methods which brought the images into geometric alignment. The comparison of the images, location of mutual differences, and the evaluation of the conservation is described in Section 4. 


\section{PHOTOGRAPH RESTORATION}

The quality of the historical photograph is poor, many scratches and dust are clearly visible at several places. The wrong setting of the camera focus (with large format cameras of that time, it was difficult to achieve high precision of the focusing) has caused image blurring. Moreover, the aging effect (silver particles are subjected to the irreversible chemical process of diffusion) has a profound impact on certain areas of the picture where it manifests as blurring. In general, most of the components in b/w photography (fine-grained and superficial silver, gelatin, albumin or collodium as a binding agent) are affected by many environmental conditions and the stability of photographic pictures is limited by the properties of each of the mentioned substances.

The removal of these degradations on the historical mosaic photograph requires an appropriate image restoration technique which would decrease the noise impact and increase the image sharpness. Most of the image degradation processes can be, at least locally, successfully modeled by linear shift invariant system

$$
g=f * h+n
$$

where $g, f, h$, and $n$ represent the acquired degraded image, original image, point spread function (PSF) and additive noise, respectively. The operator $*$ stands for the convolution. We assume that the effects, vividly apparent on the historical photograph, can be sufficiently approximated by this model.

For the noise suppression, three different methods were applied to decrease the noise level: wavelet-based denoising with automatic noise level estimation [5], adaptive non-linear filters [9], and iterative denoising based on Mumford-Shah's functional [8]. These methods were chosen as examples of different approaches to noise reduction that simultaneously preserve edges and fine details. In our experiments, the wavelet-based denoising gave the most favorable results, see Fig. 3(a) - top right and (b) - top right.

Several methods for image sharpening were considered in the image preprocessing stage. As the first method, we have chosen an iterative reconstruction algorithm based on total variation [4]. This method belongs to the group of blind deconvolution techniques and does not require any prior knowledge of the PSF. However, blind single-channel reconstruction with no or too few constraints is an ill-posed problem (this is the case of the historical mosaic image), and therefore the obtained results were unsatisfactory. In another applied approach, a PSF estimation preceded the image sharpening. The partial differential equation known as the heat equation well describes the chemical process of diffusion that contributes to the overall blurring effect. It is the well-known fact that the solution of the heat equation is the convolution of the original with a Gaussian function. The inverse heat equation could be applied to reverse the effect of the diffusion process. The main drawbacks of this method are its low stability and the necessity of extra denoising at the end of the processing. Once we accept the assumption that blur can be modeled by a Gaussian function, one can use, after estimating its variance, classical non-blind deconvolution techniques like e.g. Wiener filter (see Fig. $3(a)$ - bottom left and $(b)$ - bottom left) or constrained least square methods.

The most satisfactory results were obtained by means of the total variation (TV) based reconstruction method mentioned above ([4]) but in a non-blind framework, i.e. the Gaussian function was used as the representation of the PSF. The TV norm is essentially the $L_{1}$ norm of the image gradient which is an appropriate regularization functional that allows discontinuities in functions and thus preserves edges in images. Some modifications of the TV norm are necessary to avoid difficulties associated with the nondifferentiability of the Euclidean norm at zero. This leads to the constrained minimizing problem of the following form

$$
\min _{f} \int \sqrt{|\nabla f|^{2}+\beta^{2}} \text { subject to }\|h * f-g\|^{2}=\sigma^{2}
$$

where $\sigma^{2}$ is the error (noise) level and $\beta$ is a small positive parameter. Equivalent unconstrained optimization problem can be obtained by means of Lagrange multiplier. Several linearization schemes were proposed to deal with the nonlinearity of the associated Euler-Lagrange equations: fixed point iteration scheme [11], [12], primaldual method [3] or more general half-quadratic regularization scheme proposed by Geman and Reynolds in [6]. We have followed the approach of Chambolle and Lions [2] which introduces an auxiliary function and is similar to the half-quadratic regularization scheme. Fig. $3(a)$-bottom right and $(b)$-bottom right show the results achieved by this method on the chosen samples.

The results of applied restoration methods (Fig. 3) show slight but not demonstrative improvement. The methods produce images with less noise and/or with sharpened details. Each of the applied methods has its particular advantages and disadvantages. Unfortunately, the overall impression is not significantly better than the original non-enhanced data. 


\section{IMAGE REGISTRATION}

Before any effort to detect mutual differences can start, geometrical correspondence of images has to be assured. Since the images were taken from different locations of the camera, their geometry is different. After the alignment, all objects which did not change their position in the scene, will be at the same part of the images. Then, by means of simple image overlaying, we can easily identify parts, where the mosaic was changed with respect to its state in 1879.

There are various registration methods that remove geometrical differences of images (good overview is in [1]). The big differences between our images due to the violation of intensity values correspondence, blurring, scratches, noise, etc. eliminate the usage of classical correlation-like methods. Feature based methods, which use extracted features and their estimated correspondence, or up-to-date multimodal area based techniques [10] are appropriate candidates for the solution of our task.

The proposed approach combines both of the mentioned categories. Firstly, the features, i.e. salient point pairs (easily distinguishable points on both images, such as corners and edge endings), were manually selected, their correspondence was used to estimate the scale difference between images, and the images were rescaled to the same scale. 20 point pairs which were manually selected are shown in Fig. 6. In general, the feature selection is difficult task due to the differences between the images (blur, noise, intensity changes), but the accuracy of feature selection can strongly influence the quality of the resulting registration. Thus, the location of identified salient points in the historical image was improved by means of the mutual information method (MI) with Parzen windows [10]. Contrary to the classical correlation-based methods, which use directly image intensities, the MI criterion is not explicitly dependent on image function and can be thus applied to our data, which have multimodal character caused by the low sensitivity of the historical photograph to the red part of the visible spectra.

We computed the MI between the point pairs locally using the window neighborhood around the particular feature points. Positions of the shifted windows, which refer to the maximum value of the MI criterion, were regarded as the refined coordinates of the salient points in the historical image. For $75 \%$ of the pairs the MI criterion has well localized peeks, see Fig. 7, and gives us more accurate positions of feature points. The rest of point pairs have not enough distinctive neighborhoods and the MI gave misleading results which were refused.

An interesting result was obtained for the salient point shown in Fig. 8. The corresponding point in the historical image was incorrectly identified during the manual selection since the human perception automatically fills missing patterns, which was misleading in this case. On the other hand, MI method evaluates the whole region and therefore has found a more accurate position. The graph of MI criterion for this neighborhood is in Fig. 7.

The refined point positions and their correspondence were then used for the computation of the transformation model parameters. Considering the expected difference in the camera position, we applied the affine model

$$
\begin{aligned}
& u=a_{0}+a_{1} x+a_{2} y \\
& v=b_{0}+b_{1} x+b_{2} y
\end{aligned}
$$

and the projective model

$$
\begin{aligned}
& u=\frac{a_{0}+a_{1} x+a_{2} y}{1+c_{1} x+c_{2} y} \\
& v=\frac{b_{0}+b_{1} x+b_{2} y}{1+c_{1} x+c_{2} y}
\end{aligned}
$$

for the geometrical transformation of the images. Here, $(u, v)$ and $(x, y)$ are the coordinates in the original and the to-be-registered images, respectively. We tried the thin-plate spline model [7] too, to be able to eliminate possible local deformations. However, the affine transform proved to be sufficient.

The current image was transformed and resampled by means of the bilinear interpolation. Examples of the registered parts together with the historical originals are in Fig. 9.

\section{CHANGE DETECTION}

Finally, by transforming and overlaying the registered images of the historical and current mosaic state, many differences, not visible before, became apparent. Examples of the identified differences can be seen in Fig. 9, where two rectangular parts of the mosaic are presented. In the Resurrection scene, examples of the differences are following: the haircut of the figure has an extra wave (difference No.1), the ornamental patterns on the right 
side are corrupted and have a different shape now (difference No.2), a part of the coffin edge is missing (difference No.3). In the Jesus Christ scene, the rays under Jesus Christ are missing (difference No.1), the shape of Jesus' beard is changed (difference No.2) and the collar of one of the angels is different (difference No.3), to name some.

The task was very difficult due to the low image quality and missing color information on the historical photograph, however, we were able to identify differences, which could bring new ideas to the understanding of the mosaic and which were appreciated by art historians.

\section{CONCLUSION}

The fast development of computers and algorithms during last decades can influence areas as distant to computer technology as art conservation is. The aim of conservators, saving the heritage of the past for the next generations, can be facilitated using the high-tech devices and methods.

Our task was to use modern image processing methods for the evaluation of the conservation, realized on the medieval mosaic "The Last Judgment". We were asked to compare the historical photograph from the end of the $19^{\text {th }}$ century, very much destroyed by aging, noise, and blurring, with the digital photograph of the mosaic current state. The main aim was to identify differences and to affirm patterns, which were not changed. Moreover this experiment was the test case whether digital image processing can be of any use for the conservation of art pieces of this kind.

We applied several image restoration methods to improve the quality of the historical photograph but due to the very complex nature of image degradation no impressive results were achieved. The increase of the quality is not significant, however, small improvements in denoising and sharpening have been realized.

The second part, the image registration and location of differences, gives much better results. Using the featurebased registration approach combined with the mutual information for the feature accurate localization, the geometrical deformations due to the non-corresponding positions of the cameras during the image acquisition were removed and the new differences, not apparent before, revealed.

The digital image processing substantiates itself as a useful tool, applicable in the area of art protection and conservation and able to supply information not available otherwise. However, because of the complex interdisciplinary nature of the problem, the image processing techniques applied in such cases are not likely to work fully automatically. Human assistance of computer scientists as well as art historians will be always required.

\section{Acknowledgment}

This work has been supported by Prague Castle Administration and partially by the grant No. 102/01/P065 of the Grant Agency of the Czech Republic. 

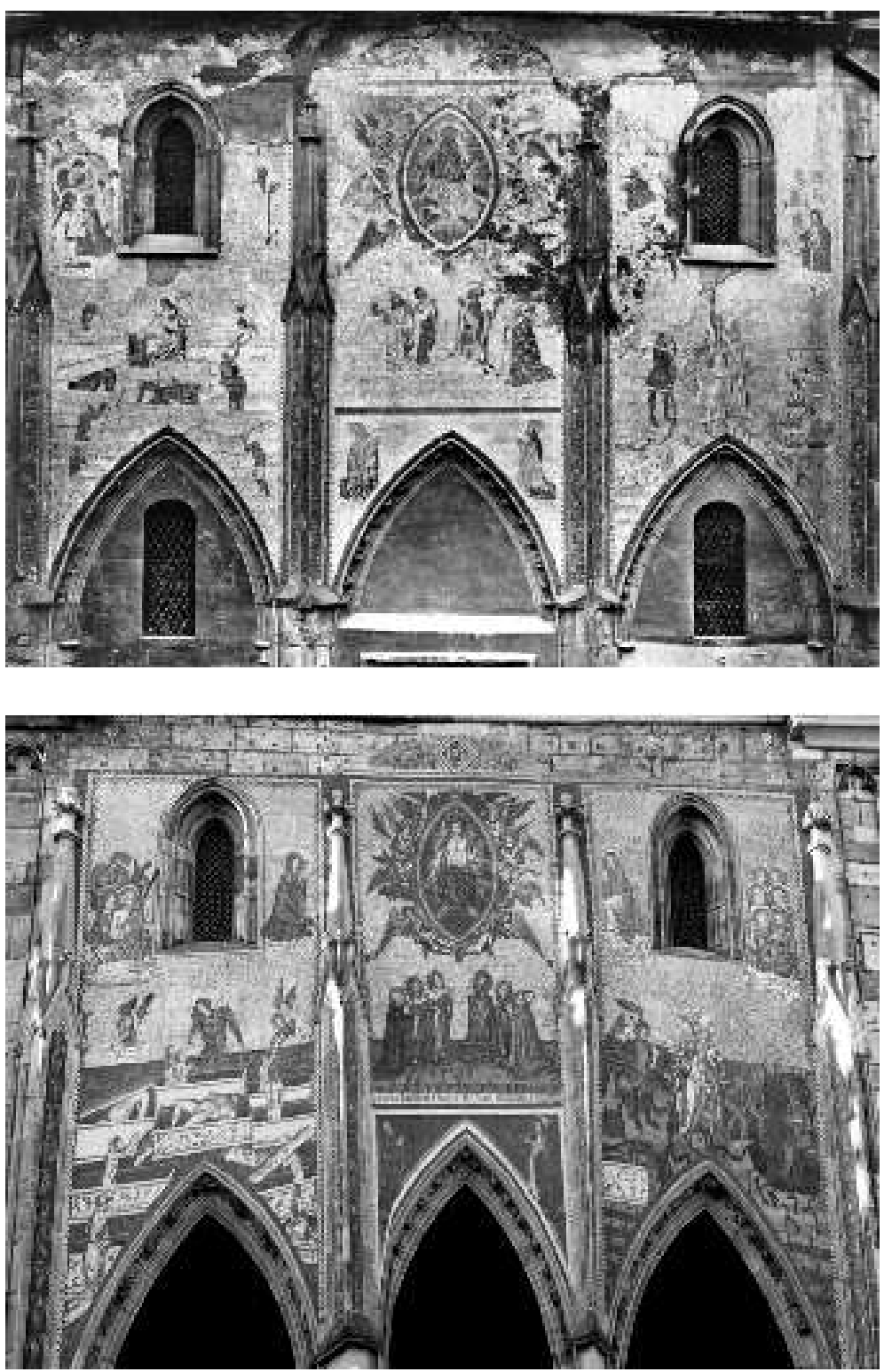

Figure 1: The Last Judgment Mosaic, situated on the outer wall of the St.Vitus cathedral. Top: The historical photograph of the mosaic by Jindřich Eckert, 1879. Bottom: The photograph of the current state taken by digital camera.

\section{References}

[1] L. G. Brown. A survey of image registration techniques. ACM Computing Surveys, 24(4):326-376, December 1992.

[2] A. Chambolle and P.L. Lions. Image recovery via total variation minimization and related problems. Numer. Math., 76(2):167-188, April 1997.

[3] T.F. Chan, G.H. Golub, and P. Mulet. A nonlinear primal-dual method for total variation-based image restoration. SIAM J. Sci. Comput., 20(6):1964-1977, July 1999.

[4] T.F. Chan and C.K. Wong. Total variation blind deconvolution. IEEE Trans. Image Processing, 7(3):370-375, March 1998.

[5] D.L. Donoho. De-noising by soft-thresholding. IEEE Trans. Inform. Theory, 41(3):613-627, May 1995. 


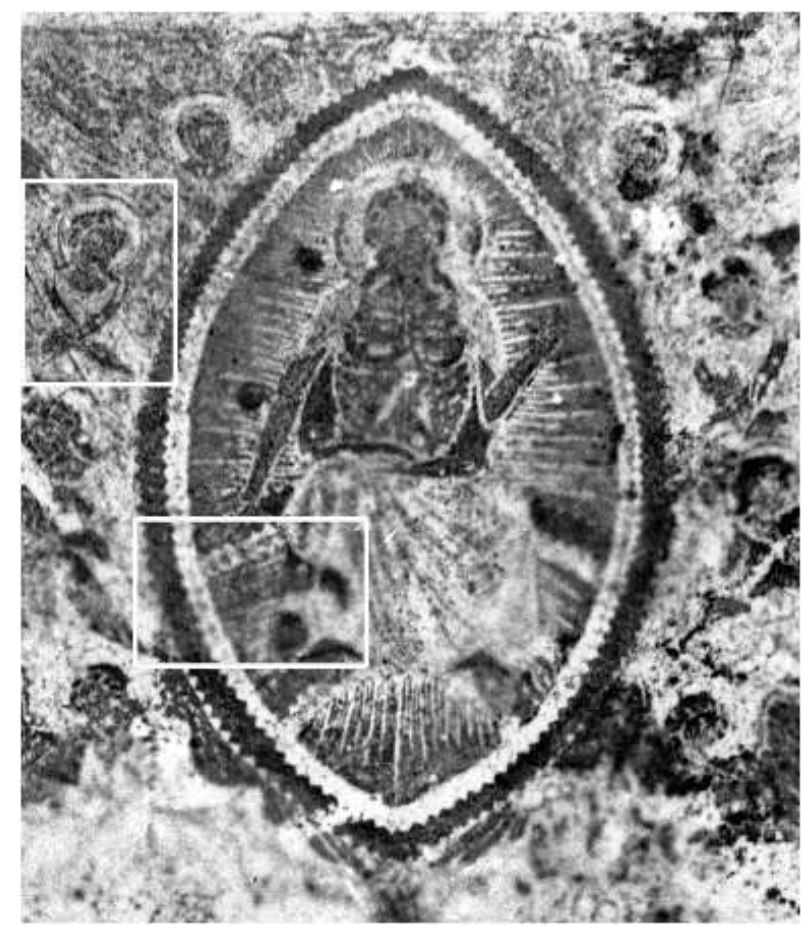

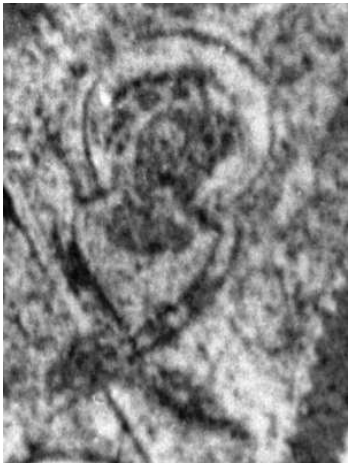

(a)

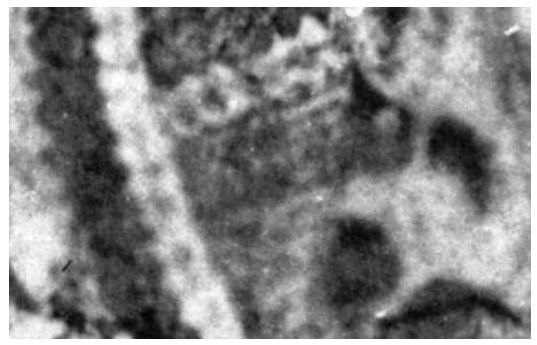

(b)

Figure 2: Two rectangular areas (see the rectangles in the left image), of size (a): $300 \times 400$, (b): $450 \times 300$, which were selected as samples for further investigation.

[6] D. Geman and G. Reynolds. Constrained restoration and the recovery of discontinuities. IEEE Trans. Pattern Anal., 14(3):367-383, March 1992.

[7] A. Goshtasby. Registration of images with geometric distortions. IEEE Transactions on Geoscience and Remote Sensing, 26(1):60-64, January 1988.

[8] D. Mumford and J. Shah. Optimal approximation by piecewise smooth functions and associated variational problems. Comm. Pure Appl. Math., 42:577-685, 1989.

[9] S. M. Smith and J. M. Brady. Susan - a new approach to low level image processing. International Journal of Computer Vision, 23(1):45-78, May 1997.

[10] P. Viola and W. M. Wells. Alignment by maximization of mutual information. International Journal of Computer Vision, 24(2):137-154, 1997.

[11] C.R. Vogel and M.E. Oman. Iterative methods for total variation denoising. SIAM J. Sci. Comput., 17(1):227238, January 1996.

[12] C.R. Vogel and M.E. Oman. Fast, robust total variation-based reconstruction of noisy, blurred images. IEEE Trans. Image Processing, 7(6):813-824, June 1998. 


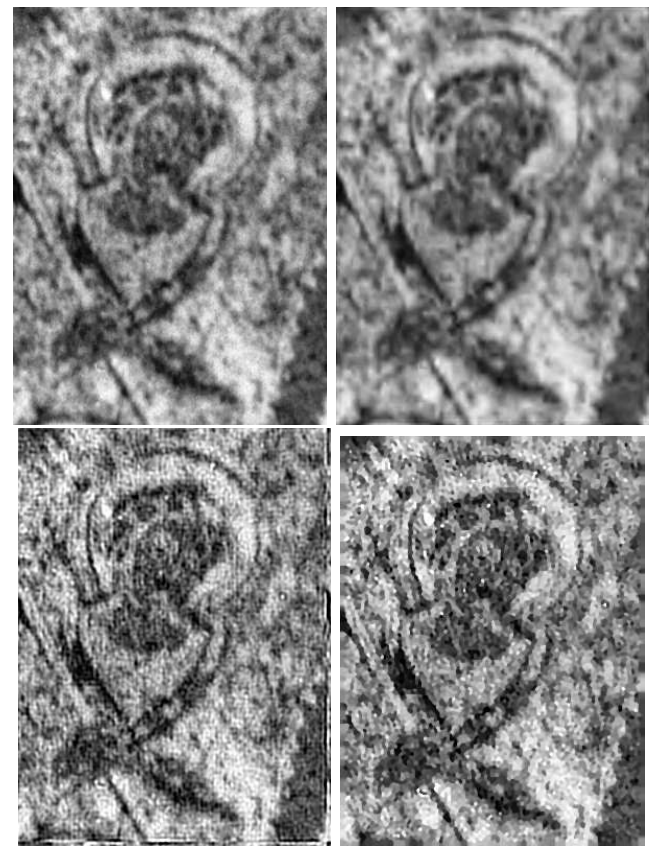

Sample (a)
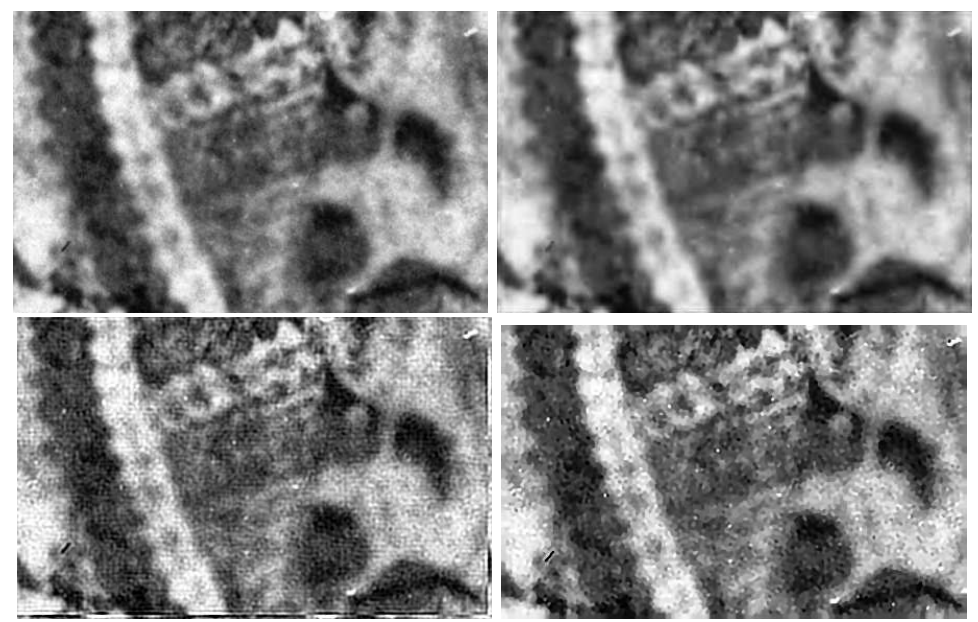

Sample (b)

Figure 3: Results of the historical photograph restoration (samples (a) and (b)): original - (top left); waveletbased denoising - (top right); Wiener filter sharpening and denoising - (bottom left); total variation sharpening and denoising - (bottom right). 


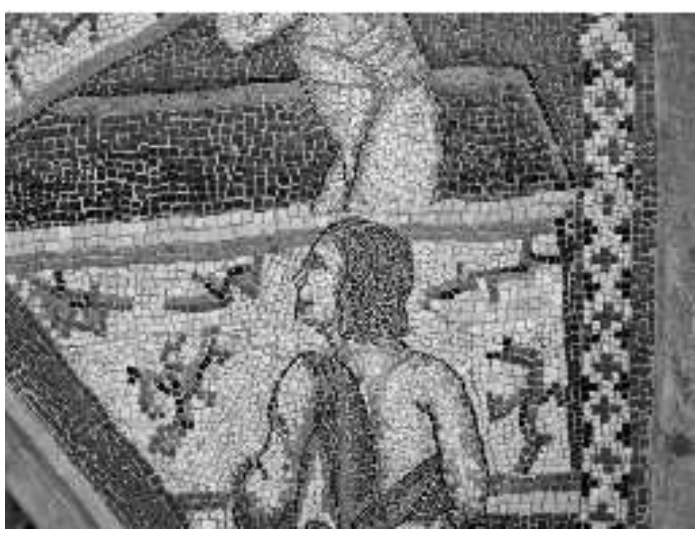

(a)

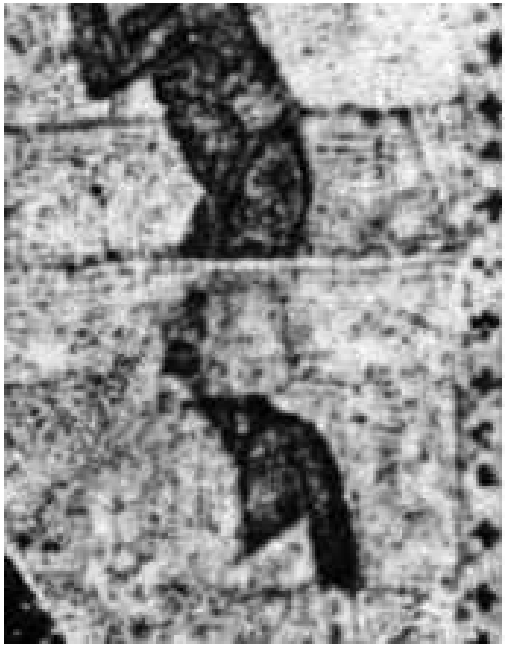

(b)

Figure 4: The Resurrection scene, used for the image alignment and the change detection: the current photograph (a), the historical photograph (b).

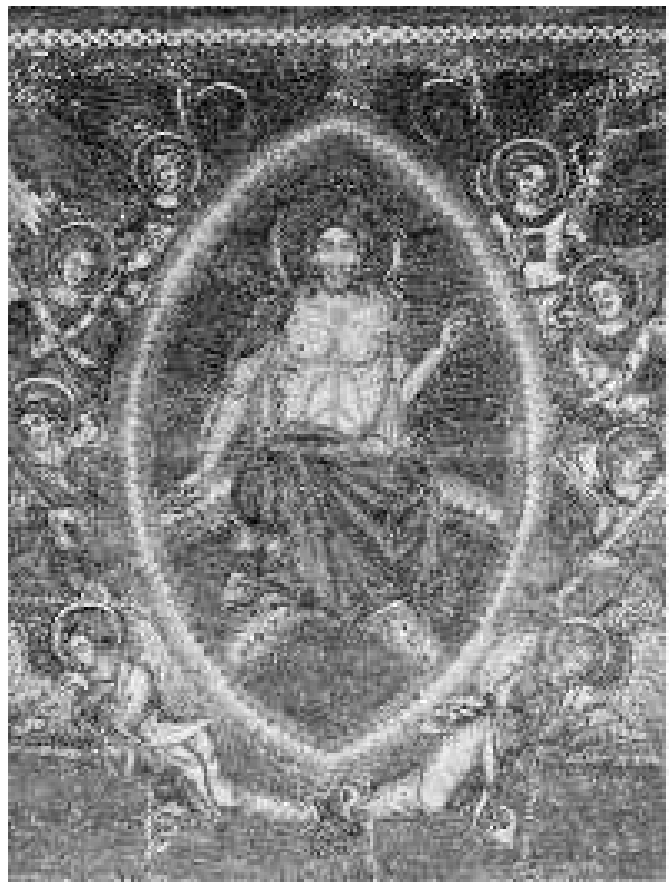

(a)

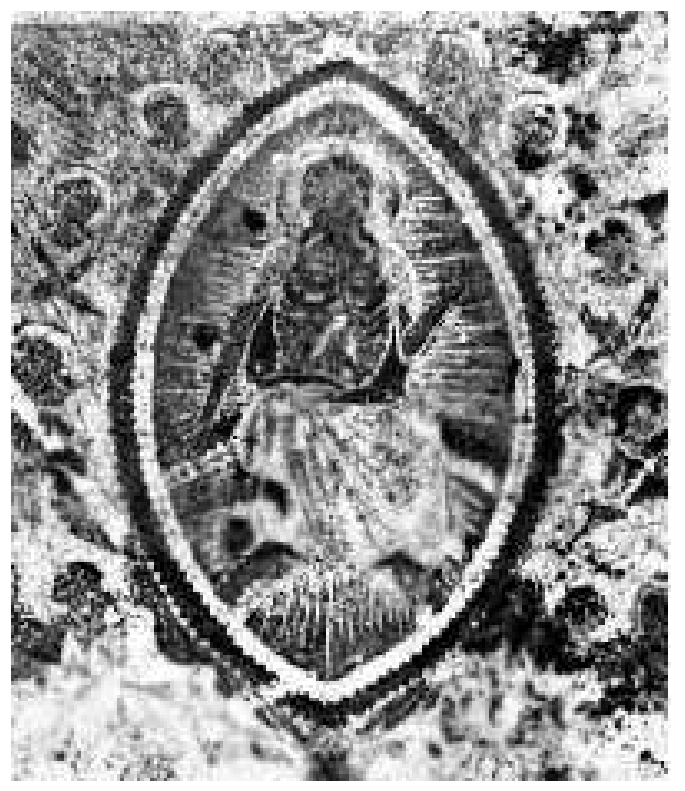

(b)

Figure 5: The Jesus Christ scene, used for the image alignment and the change detection: the current photograph (a) the historical photograph (b). 


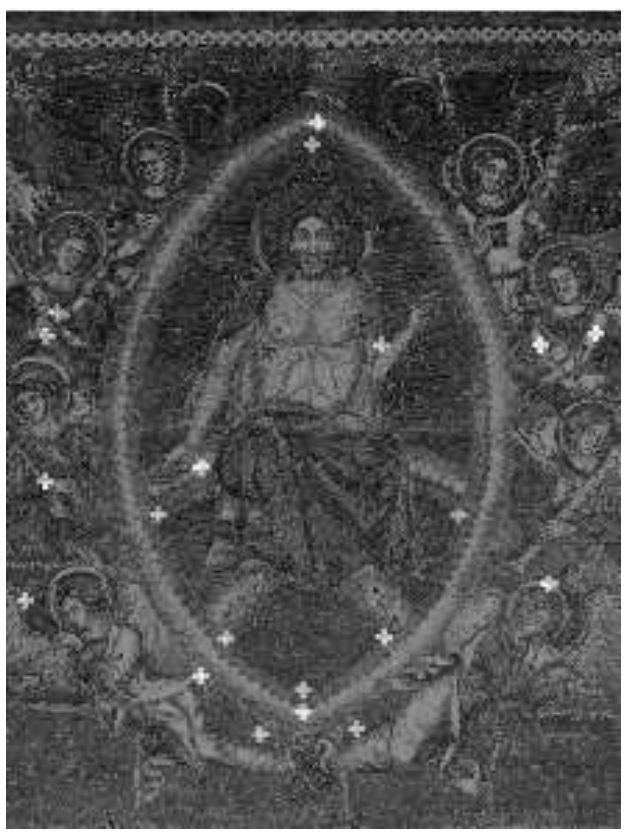

(a)

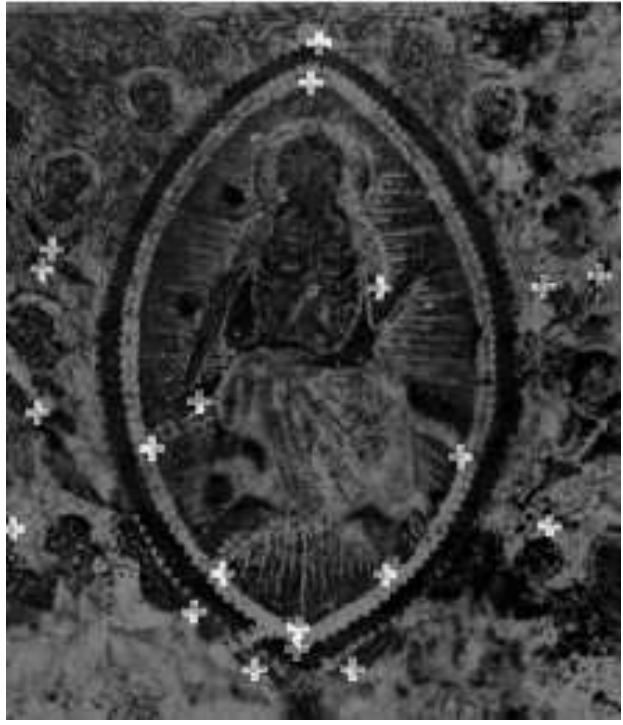

(b)

Figure 6: Selected features - point pairs. Their positions are marked by crosses. The Jesus Christ scene, the current (a) and the historical (b) images.
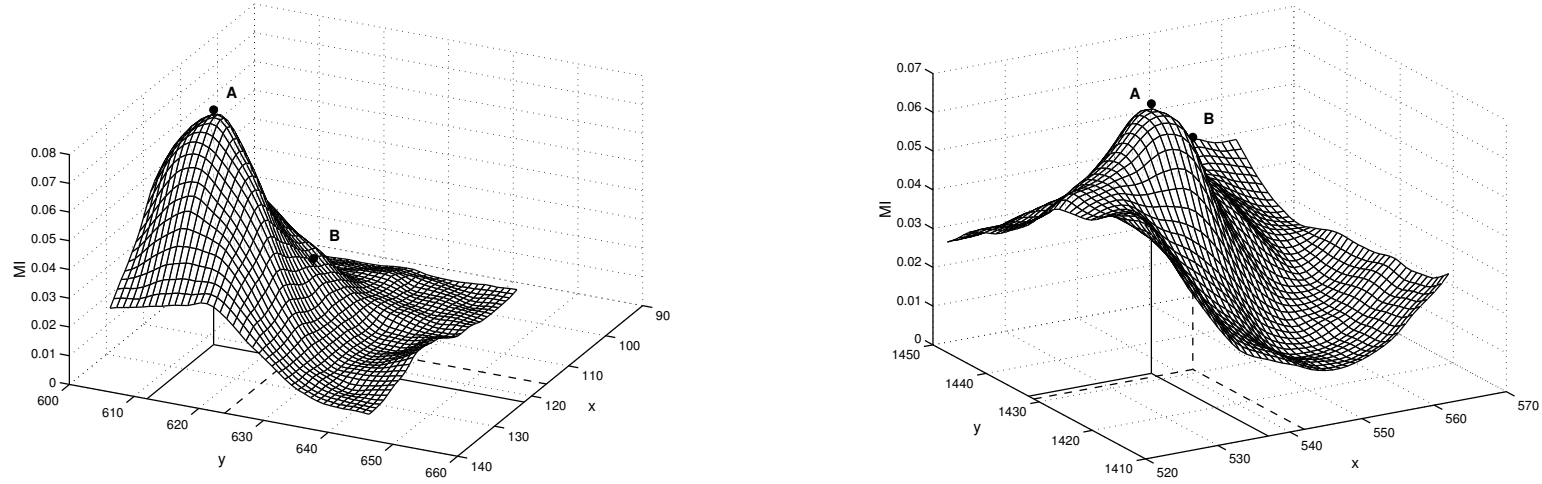

Figure 7: The mutual information criterion for two different point pairs. A represents the improved position of the point (the local maxima of MI), B marks the original, manually selected point in the historical image. The graph on the right-hand side was computed for the point pair shown in Fig. 8. The difference in the location is 13 pixels. 

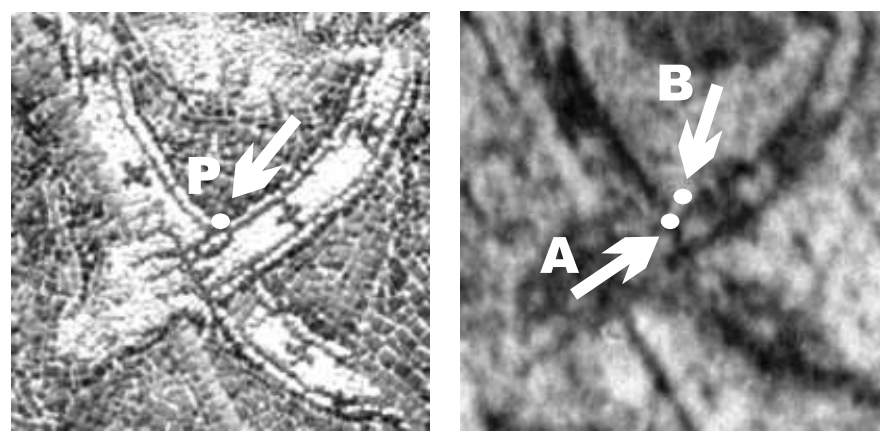

Figure 8: The improvement of the feature localization. In the historical image, the position of $\mathbf{A}$, the counterpart to the $\mathbf{P}$ point in the current image, was improved by means of the MI method. The more accurate location is marked by $\mathbf{B}$. The person, who selected the points, misjudged the shape of the straps, which was changed during the mosaic conservations.

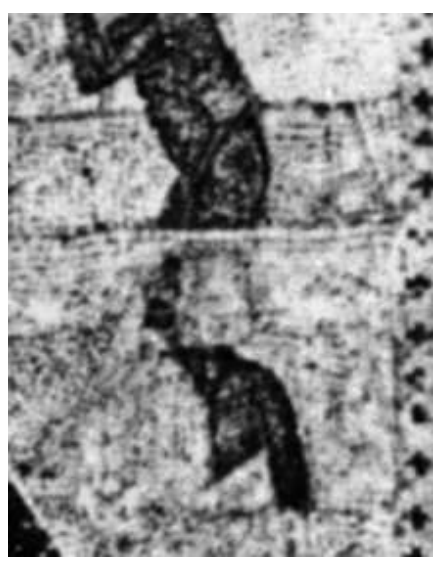

Historical image - Resurrection scene

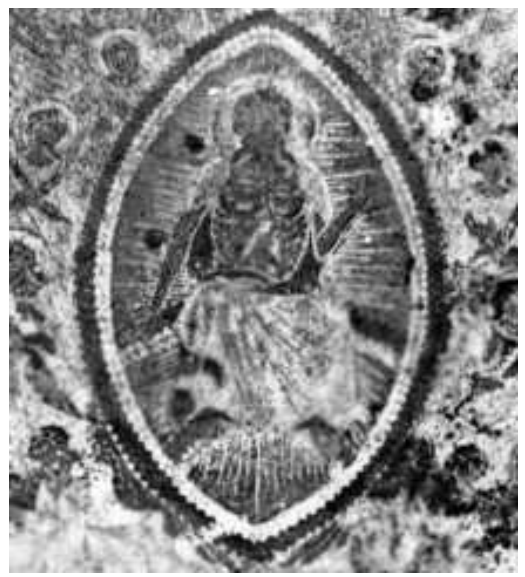

Historical image - Jesus Christ scene

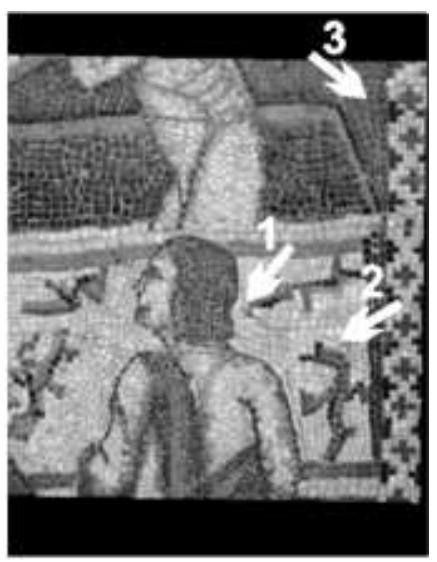

Registered current image

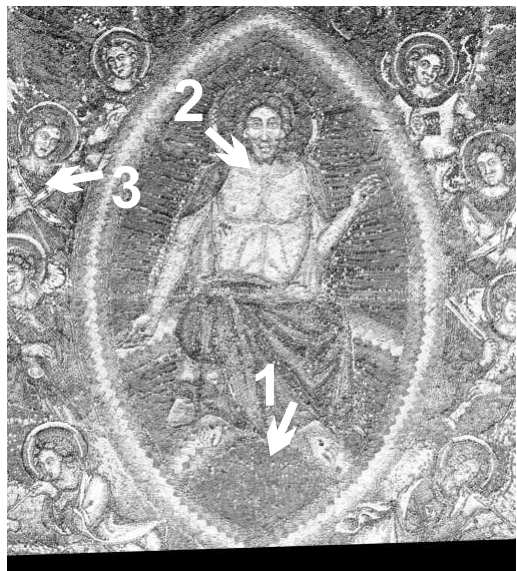

Registered current image

Figure 9: Examples of identified differences between the registered images (Resurrection and Jesus Christ scenes). Resurrection scene (top row): (1) haircut, (2) ornamental patterns, (3) coffin edge; Jesus Christ scene (bottom row): (1) rays, (2) beard, (3) collar. 